\title{
Infusion of clinical-grade enriched regulatory $T$ cells delays experimental xenogeneic graft-versus-host disease
}

\author{
Muriel Hannon, Chantal Lechanteur, Sophie Lucas, Joan Somja, Laurence Seidel, Ludovic Belle, \\ France Bruck, Etienne Baudoux, Olivier Giet, Anne-Marie Chantillon, Philippe Delvenne, \\ Pierre Drion, Yves Beguin, Stéphanie Humblet-Baron, * and Frédéric Baron*
}

BACKGROUND: We investigated the ability of clinicalgrade enriched human regulatory $T$ cells (Treg) to attenuate experimental xenogeneic graft-versus-host disease (GVHD) induced by peripheral blood mononuclear cells (PBMNCs; autologous to Treg) infusion in NSG mice, as well as verified their inability to induce xenogeneic GVHD when infused alone.

STUDY DESIGN AND METHODS: Human Treg were isolated from peripheral blood apheresis products with a cell separation system (CliniMACS, Miltenyi Biotec $\mathrm{GmbH}$ ) using a two-step procedure (simultaneous CD8 and CD19 depletion followed by CD25-positive selection) in six independent experiments with six different healthy volunteer donors. Sublethally (2.5 Gy) irradiated NSG mice were given $2 \times 10^{6}$ cytapheresis (PBMNC) product cells intravenously (IV) without (PBMNC group) or with $1 \times 10^{6}$ Treg (PBMNC + Treg group), while other NSG mice received $2 \times 10^{6}$ enriched Treg alone (also in IV; Treg group).

RESULTS: The first five procedures were successful at obtaining a relatively pure Treg population (defined as $>50 \%$ ), while the sixth procedure, due to a technical problem, was not (Treg purity, 42\%). Treg cotransfusion significantly delayed death from xenogeneic GVHD in the first five experiments, $(p<0.0001)$ but not in the sixth experiment. Importantly, none of the mice given enriched Treg alone (Treg group) experienced clinical signs of GVHD, while, interestingly, the CD4+ cells found in these mice 26 days after transplantation were mainly conventional T cells (median CD25+FoxP3+ cells among human CD4+ total cells were only 2.1, 3.1, and $12.2 \%$ in spleen, marrow, and blood, respectively). CONCLUSIONS: Infusion of clinical-grade enriched Treg delayed the occurrence of xenogeneic GVHD without inducing toxicity in this murine model.
A

llogeneic hematopoietic cell transplantation (allo-HCT) has been a potentially curative option for many patients with hematologic mancies, acquired aplastic anemia, and selected inherited blood or immune disorders. In patients with hematologic malignancies, allo-HCT has been initially designed as a way to administer supralethal doses of total body irradiation (TBI). ${ }^{1}$ However, an important part of the efficacy of the procedure is mediated by the destruction of recipient tumor cells by donor $T$ cells present in the graft (graft-versus-tumor effects). ${ }^{2-5}$ Unfortunately, donor immune cells present in the graft can also target recipient

ABBREVIATIONS: allo-HCT = allogeneic hematopoietic cell transplantation; $\mathrm{APC}=$ allophycocyanin; $\mathrm{BM}=$ bone marrow; $\mathrm{HR}=$ hazard ratio; $\mathrm{PB}=$ peripheral blood; $\mathrm{TBI}=$ total body irradiation; TREC(s) = T-cell receptor excision circle(s); Treg = regulatory $\mathrm{T}$ cells.

From the Groupe Interdisciplinaire de Génoprotéomique Appliquée (GIGA)- $\mathrm{I}^{3}$, the Laboratory of Cell and Genetic Therapy, Department of Pathology, the Department of Statistics, GIGA-R, and the Department of Medicine, Division of Hematology, University of Liège, Liège, Belgium; de Duve Institute, Université Catholique de Louvain, Brussels, Belgium; the Red Cross Transfusion Center of Liege, Liège, Belgium; and the Autoimmune Genetics Laboratory, University of Leuven, Leuven, Belgium.

Address reprint requests to: Frédéric Baron, Department of Hematology, University of Liège, CHU Sart-Tilman, 4000 Liège, Belgium; e-mail: f.baron@ulg.ac.be.

${ }^{*} \mathrm{FB}$ and SHB are co-senior authors.

This study was supported by funds from the National Fund for Scientific Research (FNRS), the Leon Fredericq fund and Anti-Cancer Center at the University of Liège, and the Belgian Federation Against Cancer. MH and LB are Télévie Research Assistant, SHB is postdoctoral researcher, and SL and FB are senior research associates of the National Fund for Scientific Research (FNRS) Belgium.

Received for publication February 26, 2013; revision received April 18, 2013, and accepted April 23, 2013.

doi: $10.1111 /$ trf. 12279

TRANSFUSION 2014;54:353-363. 
tissues, causing graft-versus-host disease (GVHD), a lifethreatening complication of allo-HCT. ${ }^{6,7}$ The first year after allo-HCT, establishment of graft-versus-host tolerance has been successful in $30 \%$ to $70 \%$ of the patients given unmanipulated grafts from HLA-matched donors, while the remaining patients experience chronic GVHD, ${ }^{8,9}$ It has been recognized since the late 1970s that tolerance after allogeneic allo-HCT was largely due to "a suppressor cell population" responsible for preventing GVHD by blocking sensitization of donor T cells against the host. ${ }^{10}$

Regulatory T cells (Treg) play a critical role in the maintenance of tolerance to self-antigens. ${ }^{11,12}$ Their development and function require transcription factor FOXP3. In humans, mutations in the FOXP3 gene cause Treg deficiency, and a fatal disorder characterized by immune dysregulation, polyendocrinopathy, enteropathy, and X-linked inheritance (IPEX).$^{13}$ In the past decade, much attention has been paid to the potential role of Treg after allo-HCT. In murine experimental GVHD models, administration of high doses of Treg at the time of transplantation prevented acute GVHD without apparently impairing graft-versus-tumor effects. ${ }^{14-16}$ Because thymic generation of Treg was markedly impaired, the majority of Treg the first month after allo-HCT in humans originated from expansion of mature Treg contained in the graft and exhibited an activated or memory phenotype. ${ }^{17}$ In addition, Treg from patients with extensive chronic GVHD had low telomerase activity, suggesting that failure to activate Treg telomerase activity after allo-HCT could restrict their proliferative capacity and increase their susceptibility to apoptosis, resulting in low Treg number and the development of chronic GVHD. ${ }^{18}$ These observations are in line with studies demonstrating a relative Treg deficit in the gut (but not in the stomach ${ }^{19}$ ) of patients with GVHD compared to patients without $\mathrm{GVHD}^{20}$ and an association between low Treg numbers and acute and/or chronic GVHD. ${ }^{21}$ In addition, Treg infusion allowed safe infusion of an otherwise lethal dose of conventional $\mathrm{T}$ cells in the haploidentical allo-HCT setting ${ }^{22}$ and possibly decreased acute GVHD in adult patients given double unrelated cord blood transplantation after reduced-intensity conditioning. ${ }^{23}$

We and others have recently developed a model of xenogeneic GVHD, by infusing human peripheral blood mononuclear cells (PBMNCs) into NOD-scid interleukin (IL)-2R $\gamma^{\text {null }}$ (NSG) mice. ${ }^{24}$ In that model, the severity of GVHD was closely correlated with the dose of PBMNCs infused. Here, we investigated the impact of infusion of clinical-grade enriched Treg on the development of xenogeneic GVHD in NSG mice.

\section{MATERIALS AND METHODS}

\section{PBMNC collection and Treg enrichment}

Before PBMNC collection, each volunteer donor $(n=6)$ underwent medical evaluation to assess his or her ability to donate and to explain the apheresis procedure. Each donor signed written informed consent (previously approved by the ethics committee of the CHU of Liege) for undergoing a leukapheresis for research purposes according to the standard operating procedures of the Laboratory of Cell and Gene Therapy. Leukapheresis procedures were performed using peripheral catheters and a continuous-flow blood cell separator (Cobe Spectra, Terumo BCT, Lakewood, $\mathrm{CO}$ ) and according to a MNC collection protocol. The processed blood volume was approximately 2 total blood volumes. Anticoagulation was performed with ACD-A (ratio 1:12).

Treg were isolated using a system for clinical-gradeconform large-scale cell separations (CliniMACS system, Miltenyi Biotec $\mathrm{GmbH}$, Bergisch Gladbach, Germany). Clinical-grade reagents were used. CD4+CD25+ Treg were isolated in a two-phase procedure: simultaneous CD8 and CD19 depletion followed by CD25-positive selection. In the first phase, cells were washed, adjusted to $87.5 \mathrm{~mL}$ in phosphate-buffered saline (PBS)-ethylenediaminetetraacetic acid (EDTA)-0.5\% human serum albumin (HSA), labeled with CliniMACS CD8 and CD19 reagents (Miltenyi Biotec) for 30 minutes at room temperature on an orbital shaker, rewashed, and resuspended in 100 to $300 \mathrm{~mL}$ of PBS-EDTA-0.5\% HSA (concentration on the CliniMACS had to be below $4 \times 10^{8}$ cells $/ \mathrm{mL}$ ). CD8- and CD19-labeled cells were depleted by the 2.1 depletion program of the CliniMACS instrument (Miltenyi Biotec). After being washed, the $\mathrm{CD} 8^{\text {neg }} \mathrm{CD} 19^{\text {neg }}$ cell fraction was suspended in $380 \mathrm{~mL}$ of PBS-EDTA-0.5\% HSA, labeled with CD25 reagents (Miltenyi Biotec) for 15 minutes at $4^{\circ} \mathrm{C}$ on an orbital shaker, washed, and resuspended in $100 \mathrm{~mL}$ of PBS-EDTA-0.5\% HSA. CD25+ cells were isolated by automatic positive-selection cycles using the 3.1 enrichment program on the CliniMACS ${ }^{\text {plus }}$ device. Aliquots before and after each labeling, depletion, and enrichment step were immunophenotyped.

\section{Flow cytometry analyses for Treg enrichment}

Analysis of cell surface molecules was performed by multicolor staining at each step of the cell separation process using the CliniMACS ${ }^{\text {plus }}$ system (first step of double CD8/ CD19 depletion-apheresis product, start product before CD8/CD19 depletion, discarded and depleted fractions; second step of CD25-positive selection-start fraction, positive [Treg-enriched] and negative fractions). The following antibodies specific for human cell surface antigens were used: CD45-VioBlue, CD8-allophycocyanin (APC), CD15-phycoerythrin (PE), CD14-PE, CD20-fluorescein isothiocyanate (FITC), Treg detection kit I (CD4-FITC, CD25-APC, CD127-PE), Treg detection kit II (CD4-FITC, CD25-PE, CD45-VioBlue, FoxP3-APC), all from Miltenyi Biotec. Flow cytometric data were acquired on a flow cytometer (MACSQuant analyzer, Miltenyi Biotec) and 
analyzed with software (MACSQuantify, Miltenyi Biotec). A first gate was made on CD45+ cells, a second gate on forward scatter-side scatter to eliminate cell debris, and a third gate on viable cells. The percentage of Treg was then determined as the percentage of cells with either CD4+CD25+FoxP3+ or CD4+CD25+CD127 low phenotype.

The start and Treg-enriched fractions were further phenotyped by multicolor staining. The following antibodies specific for human cell surface antigens were used: PercP-conjugated anti-CD4 (SK3 clone, Becton Dickinson, Franklin Lakes, NJ); PE-conjugated anti-CD25 (4E3 clone, Miltenyi Biotec); biotin-conjugated antiCD127 (RDR5 clone, eBioscience, San Diego, CA), antiCCR6 (11A9 clone, Becton Dickinson), anti-CCR5 (T21/8 clone, eBioscience) or anti-CCR4 (1G1 clone, Becton Dickinson); APC-eFluor 780 conjugated anti-streptavidin (eBioscience); PeCy7-conjugated anti-CCR7 (3D12 clone, Becton Dickinson); and PE-conjugated anti-CD45RA (HI100 clone, eBioscience). Two million cells were incubated for 20 minutes at $4^{\circ} \mathrm{C}$ in the dark with different mix of antibodies and then washed twice with PBS-3\% FBS (Sigma Aldrich, St Louis, MO). This process was repeated for a second 15-minute period for the streptavidin staining step. Human intracellular Foxp3 staining kit containing AlexaFluor 488 anti-Foxp3 (206D clone), isotype-matched control IgG, and cell fixation and permeabilization solution (Fix/Perm) were purchased from BioLegend (San Diego, CA). Foxp3 intracellular staining was performed according to the manufacturer's instructions. Data were acquired on a flow cytometer (FACSCanto II, Becton Dickinson) and analyzed with software (Flowjo 7.0, Tree Star, Inc., San Carlos, CA). Expression of chemokine receptors (CCR4, CCR5, and CCR6) was evaluated on both CD4+FoxP3+CD45RA+ and CD4+FoxP3+CD45RATreg.

\section{Suppression assay}

For the last two selection procedures, Treg function was assessed with a 7-hour flow cytometric assay, using suppression by Treg of the activation markers CD69 and CD154 on responder T cells (CD4+CD25-; activated with anti-CD3/CD28-coated beads [Dynabeads, Invitrogen, Gent, Belgium] at a bead/responder T-cell ratio of 0.2), as described by Canavan and coworkers. ${ }^{25}$

\section{Signal-joint T-cell receptor excision circles}

Naive (CD4+CD25+CD127-CD45RA+CCR7+) and memory (CD4+CD25+CD127-CD45RA-) Treg were isolated from the Treg-enriched fraction of Donor 5 by a cell sorter (FACS ARIA II, Becton Dickinson). Quantification of signal-joint T-cell receptor excision circles among naive and memory Treg was assessed as previously described. ${ }^{26,27}$

\section{Assessment of demethylated FOXP3il sequences}

Genomic DNA was prepared from the enriched Treg fractions with the PureLink genomic DNA mini kit (Invitrogen). One microgram of genomic DNA was treated with sodium bisulfite using a bisulfite conversion kit (EpiTect, Qiagen, Düsseldorf, Germany). Real-time polymerase chain reaction (PCR) amplification of methylated and demethylated FOXP3i1 sequences was performed on $62.5 \mathrm{ng}$ of bisulfite-converted DNA as previously described, ${ }^{28}$ with methyl-specific primers listed by Lucas and coworkers. ${ }^{29}$ The proportion of cells with demethylated FOXP3il was calculated as follows: (number of demethylated FOXP3il sequences/(number of demethylated FOXPil sequences + number of methylated FOXP3il sequences)) $\times$ number of $\mathrm{X}$ chromosomes per cell. The calculated proportions are approximate, because the methylation-specific quantitative PCR assay detects only sequences that are either completely demethylated or completely methylated in the FOXP3i1 region.

\section{Induction of xenogeneic GVHD in NSG mice}

All experimental procedures and protocols used in this investigation were reviewed and approved by the Institutional Animal Care and Use Ethics Committee of the University of Liège, Belgium (Permit 711). The "Guide for the Care and Use of Laboratory Animals," prepared by the Institute of Laboratory Animal Resources, National Research Council, and published by the National Academy Press, was followed carefully. NSG mice (The Jackson Laboratory, Bar Harbor, ME) were given 2.5 Gy TBI using a ${ }^{137} \mathrm{Cs}$ source 1 day before IV injection of either $2 \times 10^{6}$ human PBMNCs (apheresis product, PBMNC group) alone, IV injection of $2 \times 10^{6}$ human PBMNCs (apheresis product) plus $1 \times 10^{6} \mathrm{CD} 4+\mathrm{CD} 25$ enriched Treg (PBMNC + Treg group), or IV injection of $2 \times 10^{6}$ enriched Treg alone (Treg group). Each group included from five to seven mice depending on the number of NSG mice available. All cell products were infused fresh. In the first experiment, two doses of enriched Treg were tested $\left(0.5 \times 10^{6}\right.$ and $1 \times 10^{6} \mathrm{CD} 4+\mathrm{CD} 25$ enriched Treg). In the fourth experiment, all cell doses were doubled. In Experiments 2 through 6 , one mouse in each of the three groups (PBMNCs only, PBMNCs + Treg, and Treg only) was selected before transplant to be sacrificed around Day 26 (range, Days 25-27) to allow flow cytometric analyses. In Experiment 4, all mice in the PBMNC-only and the PBMNC + Treg groups died before Day 26 (and thus no mouse could be euthanized on Day 26). Further, in Experiments 3 and 6, the mice in the PBMNC group scheduled for necropsy on Day 26 died earlier and were then replaced randomly by two other PBMNC mice. Mice who suffered from terminal stage of xenogeneic GVHD were euthanized. 


\section{Detection of human engraftment by flow cytometry}

At the time of necropsy, peripheral blood (PB), spleen, and bone marrow (BM) were harvested and analyzed by flow cytometry. Splenocytes were obtained by crushing the spleen, and BM cells by flushing femurs and tibiae. Cells were counted in a hematology analyzer (XS-800i, Sysmex, Hoeilaart, Belgium). The PB was depleted of red blood cells using the RBC lysis buffer (eBiosciences) according to the manufacturer's instructions. The following antibodies specific for human cell surface antigens were used: Horizon v500-conjugated anti-CD3 (SP34-2 clone, Becton Dickinson); efluor 450-conjugated antiCD4 (RPA-T4 clone, eBioscience); APCCy7-conjugated anti-CD8 (SK1 clone, Becton Dickinson); PercPconjugated anti-CD45 (2D1 clone, Becton Dickinson); PE-conjugated anti-CD25 (BC96 clone, eBioscience), PeCy7-conjugated anti-CCR7 (3D12 clone, Becton Dickinson), APC-conjugated anti-CD45RA (HI100 clone, Becton Dickinson), biotin-conjugated anti-CCR4 (1G1 clone, Becton Dickinson), and APC-eFluor 780-conjugated antistreptavidin (eBiosciences). Cells $\left(1.5 \times 10^{6}-2 \times 10^{6}\right.$ cells/ sample) were incubated with antibodies or isotypematched control IgG (all MOPC 21 clone, Becton Dickinson) for 20 minutes at $4{ }^{\circ} \mathrm{C}$ in the dark. Cells were washed twice with PBS-3\% FBS (Lonza, Verviers, Belgium). Human Foxp3 staining was performed as described above. Percentage of Treg was identified based on the CD4+CD25+FoxP3+ phenotype on the total lymphocyte gate. Data were acquired on a flow cytometer (FACSCanto II, Becton Dickinson) and analyzed with software (Flowjo 7.0, Tree Star, Inc.).

\section{Histology and immunochemistry}

Lungs from mice euthanized on Days 25 to 27 after transplantation (one mouse in each group from Experiments 2, 3,5 , and 6) were harvested, washed with PBS, fixed in $10 \%$ formalin (Sigma Aldrich), and routinely processed for paraffin embedding. Five-micrometer sections were stained with hematoxylin-eosin for histologic examination. Xenogeneic GVHD in lung sections was assessed in a semiquantitative fashion as described by Nervi and colleagues. $^{30}$

\section{Statistical analyses}

The Mann-Whitney test was used to compare homing receptor expression between naive and memory Treg and between lymphocyte subsets at necropsy in mice from different groups. Survival curves were modeled using the Kaplan-Meier methods. Comparisons between groups were made with the log-rank test. The impact of Treg infusion on survival was also assessed in a multivariate Cox model including group (PBMNC vs. PBMNC + Treg) and experiment number ( 1 vs. 2 vs. 3 vs. 4 vs. 5 vs. 6 ) as cova- riates. All $\mathrm{p}$ values were two sided. $\mathrm{p}$ values less than 0.05 were considered as significant. Statistical analyses were carried out with computer software (GraphPad Prism, GraphPad Software, San Diego, CA; and SAS Version 9.2 for Windows, SAS Institute, Cary, NC).

\section{RESULTS}

\section{Selection of Treg: phenotypic features}

The initial leukapheresis product contained a median of $153 \times 10^{8}$ (range, $83 \times 10^{8}-293 \times 10^{8}$ ) viable white blood cells (WBCs). A median of $17.7 \times 10^{8}$ viable WBCs were taken from the leukapheresis product for mouse experiments and for analysis. Due to a technical problem (incomplete collection bag connection to the column) during the sixth selection procedure, $31 \%$ of the leukapheresis product was lost. As seen in Table 1, this technical problem dramatically impacted the purity of the selected Treg, probably because too few cells were loaded onto the column for the first and thus the second step of the procedure. Hence, the starting fraction of the six experiments contained a median of $129 \times 10^{8}$ (range, $54 \times 10^{8}$ $276 \times 10^{8}$ ) viable WBCs. After the two-step selection procedure, a median of $1.8 \times 10^{8}$ (range, $0.7 \times 10^{8}$ $2.3 \times 10^{8}$ ) viable cells were recovered. The median percentage of CD4+CD25+CD127 dim/low and CD4+CD25+FoxP3+ cells in the final products were 79 (range, 52-82) and 66 (range, 42-68), respectively, with consistently lower figures in the sixth experiment. The final fraction contained virtually no monocytes, B cells, nor CD8+ T cells. Finally, we also measured the proportion of demethylated FOXP3 Intron 1 (FOXP3il) sequences. Demethylation of this regulatory region of gene FOXP3 is considered the most specific marker of human Treg, because in contrast to protein FOXP3, it is not found in activated non-Treg. The proportions of demethylated FOXP3il sequences in the Treg-enriched fractions were 42.5 and $32.4 \%$, in Experiments 5 and 6 , respectively.

We then further examined the Treg phenotype in the Treg-enriched fraction. Thirty-six percent (range, $20 \%-36 \%$ ) of Treg had a naive phenotype (defined as CD4+FoxP3+CD45RA+ $\mathrm{T}$ cells), while $64 \%$ (range, $64 \%-80 \%$ ) had a memory phenotype (defined as CD4+FoxP3+CD45RA-). To assess the maturation and migratory capacities of Treg, we also analyzed expression of homing receptors by Treg subsets. CCR4, CCR5, and CCR6 homing receptors were expressed in 16.5\% (range, 8.3\%-23.4\%), $1.7 \%$ (range, $1.1 \%-7.0 \%$ ), and $16.7 \%$ (range, 6.5\%-27.2\%) of naive Treg, versus in $88.2 \%$ (range, $83.3 \%$ $91.8 \% ; \mathrm{p}=0.008$ ), $27.5 \%$ (range, $23.6 \%-32.4 \% ; \mathrm{p}=0.008$ ), and $73.4 \%$ (range, $64.5 \%-83.5 \%$; $\mathrm{p}=0.008$ ) of memory Treg, respectively. As expected, naive Treg contained higher T-cell receptor excision circles (TRECs) amount than memory Treg ( 1967 vs. 85 TRECS $/ 10^{5}$ cells) in the products from Donor 5. 


\begin{tabular}{|c|c|c|c|c|c|c|}
\hline \multirow[b]{2}{*}{ Parameter } & \multicolumn{6}{|c|}{ Experiment } \\
\hline & 1 & 2 & 3 & 4 & 5 & $6 \dagger$ \\
\hline Donor age (years) & 44 & 31 & 34 & 38 & 29 & 26 \\
\hline \multicolumn{7}{|l|}{ Apheresis product } \\
\hline Viable WBCs $\left(\times 10^{8}\right)$ & 103 & 293 & 185 & 122 & 203 & 83 \\
\hline CD4 (\%) & NA & NA & NA & 35.3 & 42.4 & 34.1 \\
\hline CD8 $(\%)$ & NA & 21.9 & 23.5 & 21.3 & 21.7 & 24.3 \\
\hline CD4+CD25 high (\%) & NA & NA & NA & 2.4 & 3 & 2.4 \\
\hline CD4+CD25+CD127- (\%) & NA & NA & NA & 2.4 & 3.2 & 2.5 \\
\hline CD4+CD25+FoxP3+ (\%) & NA & NA & NA & 2 & 2.9 & 1.7 \\
\hline Demethylated FOXP3i1 sequences (\%) & & & & & 1.0 & 0.7 \\
\hline \multicolumn{7}{|l|}{ Start fraction } \\
\hline Viable WBCs $\left(\times 10^{8}\right)$ & 90.6 & 275.5 & 149.4 & 108 & 185 & 53.7 \\
\hline \multicolumn{7}{|l|}{ After the two-step procedure } \\
\hline Viable WBC $\left(\times 10^{8}\right)$ & 2.0 & 2.0 & 1.5 & 1.6 & 2.3 & 0.7 \\
\hline CD4 (\%) & 94.5 & 98.5 & 97.9 & 98.4 & 96.7 & 97.0 \\
\hline CD8 $(\%)$ & 0 & NA & NA & NA & 0.004 & 0.03 \\
\hline CD4+CD25 high (\%) & NA & 77.5 & 72.6 & 70.7 & 74.1 & 52.2 \\
\hline CD4+CD25+CD127- (\%) & NA & 82.5 & 79.2 & 77.3 & 80.1 & 52.3 \\
\hline CD4+CD25+FoxP3+ (\%) & $58.8 \ddagger$ & 64.7 & 67.4 & 68.2 & 67.9 & 42.4 \\
\hline$\%$ of $\mathrm{CD} 4+\mathrm{CD} 25+$ FoxP3+ recovery after the two-step procedure (yield)§ & NA & NA & NA & 44.7 & 26.5 & 21 \\
\hline Demethylated FOXP3i1 sequences (\%) & & & & & 42.5 & 32.4 \\
\hline \multicolumn{7}{|l|}{ Suppression assayף } \\
\hline CD69 (\%) & & & & & $8.5 / 27.0$ & $0.4 / 11.3$ \\
\hline CD154 (\%) & & & & & $19.0 / 49.2$ & $14.7 / 30.7$ \\
\hline \multicolumn{7}{|c|}{$\begin{array}{l}\text { * A first gate was made on CD45+ cells, a second gate on forward scatter-side scatter to eliminate cell debris, and a third gate on viable } \\
\text { cells. } \\
\text { T Technical problem. } \\
\text { ₹ CD4+FoxP3+ cells. } \\
\S \text { Somewhat underestimated because a median of } 17.7 \times 10^{8} \text { viable WBCs was taken from the leukapheresis product for mouse experi- } \\
\text { ments and for analysis; NA = not available. } \\
\text { T The two values shown in each column represent the percentage decrease (in comparison to expression in the absence of Treg) of the } \\
\text { expression of activation markers (CD69 and CD154) by CD25- T cells activated with anti-CD3/CD28-coated beads, in presence of Treg } \\
\text { with two CD25-:Treg cells ratios }(2: 1 / 1: 1) .{ }^{25}\end{array}$} \\
\hline
\end{tabular}

\section{Treg coinfusion delayed experimental xenogeneic GVHD}

As previously observed, ${ }^{24}$ the timing of GVHD occurrence varied from donor to donor (Fig. 1). Importantly, infusion of Treg-enriched cells delayed xenogeneic GVHD in the first five experiments in a Cox model adjusted for experiment number (hazard ratio [HR], 0.19; 95\% confidence interval $[\mathrm{CI}], 0.10-0.38 ; \mathrm{p}<0.0001)$. Specifically, the median survival in the PBMNC group versus the PBMNC + Treg group was 30 versus 56 days $(p=0.015)$ in Experiment 1,117 versus more than 162 days $(\mathrm{p}=0.136)$ in Experiment 2, 26 versus 70 days $(p=0.013)$ in Experiment 3,13 versus 16 days $(\mathrm{p}=0.038)$ in Experiment 4 , and 27 versus 49 days $(p=0.059)$ in Experiment 5 . In the first experiment, we also tested two ratios of PBMNCs/Treg ( $2 / 1$ and $4 / 1)$, and as shown in Fig. 1A, there was no difference in survival between the two ratios (median survival 56 days vs. 55 days). In the sixth cohort, Tregenriched coinfusion failed to delay experimental xenogeneic GVHD (the median survival was 46 days in the PBMNC group vs. 48 days [ $p=0.337$ ] in the PBMNC + Treg group). This could be related to the low purity of the Tregenriched fraction due to a technical problem (see above) that also resulted in a higher number of conventional
PBMNCs infused. Nevertheless, in the Cox model taking into account data from the six experiments, survival remained significantly higher in the PBMNC + Treg group than in the PBMNC group (HR, 0.34; 95\% CI, 0.19-0.62; $\mathrm{p}=0.0004$ ).

In Experiments 2, 3, 5, and 6 (in Experiment 4, mice died before Day 26), one mouse from each of the PBMNC, PBMNC + Treg, and Treg groups (each selected before transplantation) were euthanized on Days 25 to 27 (depending on experiments, median Day 26.5) after transplantation. There were no significant differences between the PBMNC and the PBMNC + Treg groups for infiltration by human CD45+CD3+ cells in the blood (median, $60.2 \%$ [range, $7.8 \%-64.6 \%$ ]) in the PBMNC group versus $23.4 \%$ [range, 1.6\%-43.6\%] in the PBMNC + Treg group), spleen (median, $11.9 \%$ [range, $7.3 \%-50.4 \%$ ]) in the PBMNC group versus $14.1 \%$ [range, $2 \%-28.7 \%$ ] in the PBMNC + Treg group), and BM (median, $10.7 \%$ [range, $0.3 \%-19.1 \%$ ]) in the PBMNC group versus $3.2 \%$ [range, $0.1 \%-10.9 \%$ ] in the PBMNC + Treg group). The frequency of T-cell subsets (CD4 and CD8) as well as their mitotic activity (assessed by KI67 expression) in the $\mathrm{PB}$, spleen, and $\mathrm{BM}$ were comparable in PBMNC and PBMNC + Treg mice (Fig. 2A-C). Interestingly, the frequency of Foxp3+ cells among total 
A

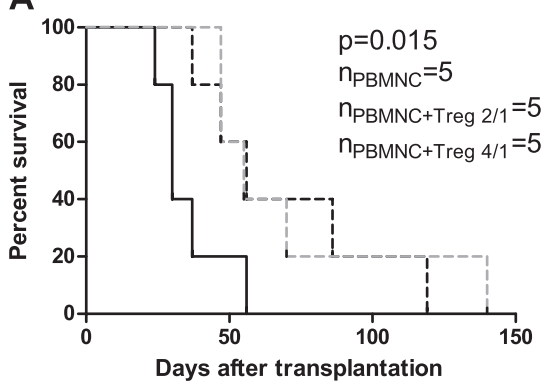

C

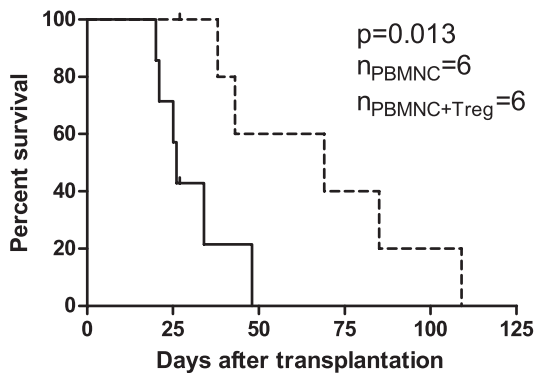

E

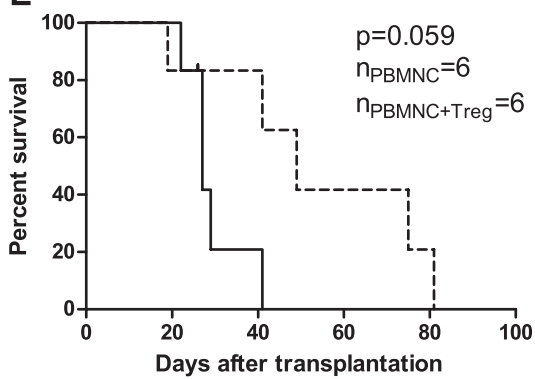

B

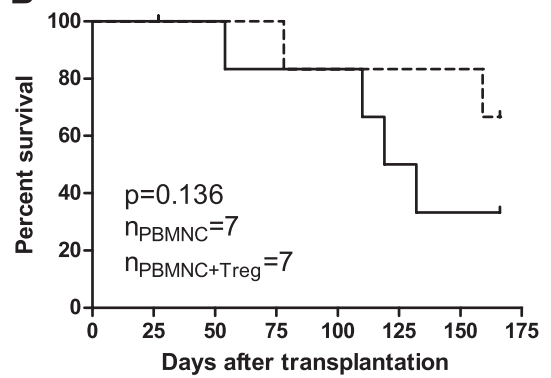

D

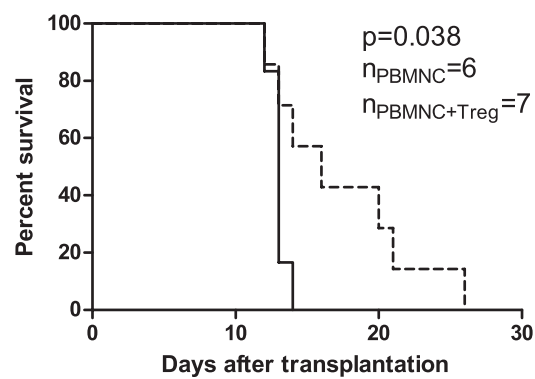

$\mathbf{F}$

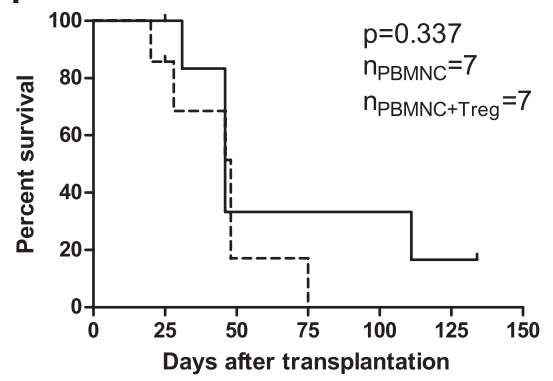

Fig. 1. Impact of Treg coinfusion on xenogeneic GVHD in NSG mice given human PBMNCs IV after 2.5 Gy TBI the day before transplantation. Continuous lines represent mice given PBMNCs only (PBMNC mice) while broken lines mice given PBMNCs and enriched Treg (Treg + PBMNC mice). Mice that were euthanized around Day 26 (Days 25-27) for flow cytometry analyses were censored on the graft. In all experiments but Experiment 4, a total of $2 \times 10^{6}$ PBMNCs were infused in PBMNC mice, while $2 \times 10^{6}$ PBMNCs $+1 \times 10^{6}$ Treg were infused in Treg + PBMNC mice. In Experiment 1, another ratio of PBMNCs and Treg (4/1) was also tested (gray broken line in Fig. 2A). For Experiment 4, all cell doses were doubled. As mentioned in the text, due to a technical problem, the purity of enriched Treg in Experiment 6 was low (42\% of CD4+CD25+FoxP3+ T cells). Graphs A through F show results in Experiments 1 through 6 , respectively. Taking data from the six experiments, survival was significantly shorter in the PBMNC group than in the PBMNC + Treg group (HR, 0.34; 95\% CI, 0.19-0.62; $p=0.0004$ ) in a Cox model adjusted for the different experiments.

human CD45+CD3+CD4+ cells was similar in the PBMNC compared to the PBMNC+ Treg groups, that is, $2.1 \%$ (range, $0.2 \%-3.1 \%$ ) versus $3.6 \%$ (range, $0.8 \%-9.5 \%$ ) in the spleen, $1.8 \%$ (range, $1.4-4.3 \%$ ) versus $0.8 \%$ (range, $0.1 \%$ $15.4 \%$ ) in the $\mathrm{BM}$, and $1.3 \%$ (range, $0.5 \%-4.5 \%$ ) versus $1.1 \%$ (range, $0.4 \%-10.8 \%$ ) in the blood (Fig. 2D). Finally, histologic signs of xenogeneic GVHD in the lungs on Day 26 were similar in the PBMNC and PBMNC + Treg groups (Table 2) and were relatively mild, probably because histology was performed relatively soon after transplantation.

\section{Fate of Treg-enriched fraction infused alone in NSG mice}

Infusion of the Treg-enriched fractions in NSG mice given 2.5 Gy TBI on Day -1 was safe with only one of 21 mice dying during the study period ( $>120$ days after transplantation) of unknown cause and none of the mice experiencing clinical GVHD (Figs. 3 and 4A; there was, however, some histologic evidence of lung GVHD in Treg mice scheduled to be euthanized on Day 26). In Experiments 2, 3, 5, and 6, one mouse (selected before transplantation) was euthanized between Days 25 and 27 (median, Day 26.5) after transplantation. At necropsy, infiltration by human CD45+CD3+, CD45+CD3+CD4+, and CD45+CD3+CD8+ cells was, respectively, $0.5 \%$ (range, $0.3 \%-8.1 \%$ ), $0.4 \%$ (range, $0.3 \%-7.9 \%$ ), and $0 \%$ (range, $0 \%$ ) in the blood; $0.6 \%$ (range, $0.4 \%$ $13.4 \%$ ), $0.2 \%$ (range, $0 \%-12.1 \%$ ), and $0.4 \%$ (range, $0.2 \%-0.7 \%$ ) in the spleen; and $0.2 \%$ (range, $0.2 \%-1.3 \%$ ), $0.2 \%$ (range, $0 \%-1.1 \%$ ), and $0 \%$ (range $0 \%-0.1 \%)$ in the BM. Interestingly, the percentage of CD25+Foxp3+ cells among total human CD45+CD3+CD4+ cells was very low, that is, $2.1 \%$ (range, $1.9 \%-12.5 \%$ ) in the spleen, $3.1 \%$ (range, $0.5 \%-3.2 \%$ ) in the $\mathrm{BM}$, and $12.2 \%$ (range, $1 \%-24.9 \%$ ) in the blood (Fig. 4B). Further, among CD4+ $\mathrm{T}$ cells in the spleen, the vast majority of CD4+ T cells were memory CD4+ $\mathrm{T}$ cells (median, 97\%; range, $84 \%-97 \%$ ).

The remaining mice were euthanized at the end of the study period (124-166 days after transplantation). Infiltrations by human CD45+CD3+, CD45+CD3+CD4+, and CD45+CD3+CD8+ cells were, respectively, $0.2 \%$ (range, $0 \%-12.6 \%$ ), $0.7 \%$ (range, $0 \%-6.5 \%$ ), and $0 \%$ (range, $0 \%-2.6 \%$ ) in the blood; $2.9 \%$ (range, $0.1 \%-51.1 \%$ ), $1.1 \%$ (range, $0 \%-34.5 \%$ ), and $0.6 \%$ (range 0\%-4.7\%) in the spleen; and 0.2\% (range, 0\%-5.6\%), $0.15 \%$ (range, $0 \%-4.4 \%$ ), and $0 \%$ (range, $0-0.7 \%$ ) in the BM. 
A

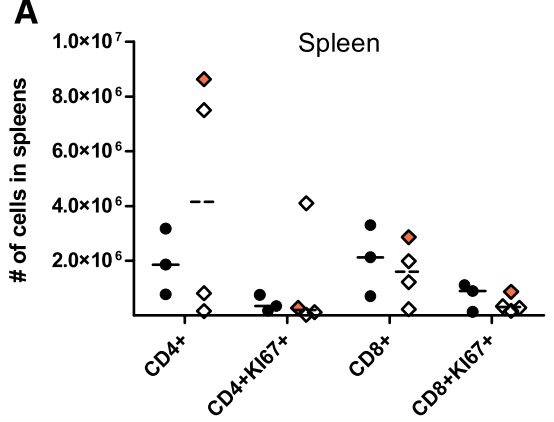

C

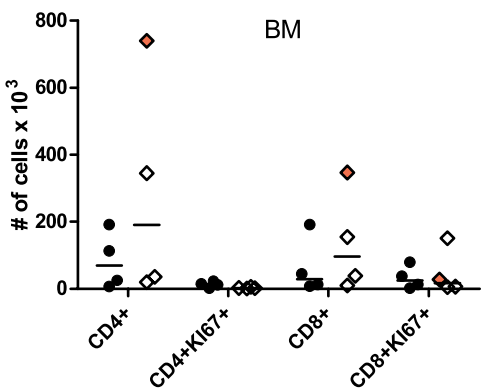

B

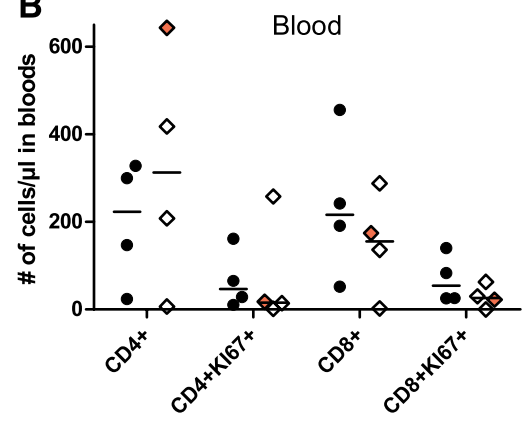

D

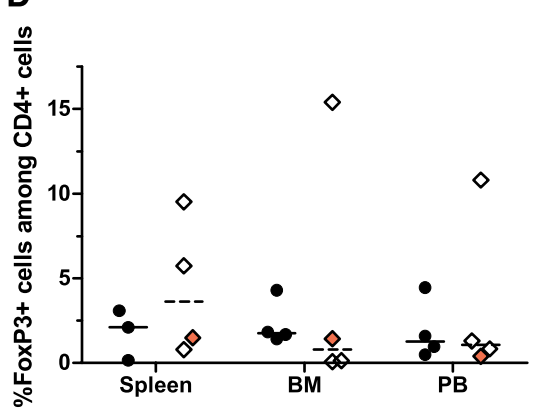

Fig. 2. (A-C) Frequency of T-cell subsets (CD4 and CD8) as well as their mitotic activity (assessed by KI67 expression) on Day 26 after transplantation in spleen (A), PB $(B)$, and BM $(C)$ of NSG mice given $(\diamond)$ or not $(\bullet)$ Treg in addition to PBMNCs. $(\diamond)$ Data from the PBMNC + Treg mouse in Experiment 6. (D) Percentage of human Foxp3+ cells among total human CD45+CD3+CD4+ cells in spleen, BM, or PB on Day 26 after transplantation in mice given $(\diamond)$ or not $(\bullet)$ Treg in addition to PBMNCs. Horizontal lines represent median values of each group. Due to a technical problem, data for the spleen of PBMNC group in Cohort 5 are missing (A and D).

\section{DISCUSSION}

Treg infusion is one of the most promising nonpharmacologic approaches for GVHD prevention or treatment. However, isolating a pure population of Treg in sufficient amount for clinical use has remained challenging (outside of the HLA-haploidentical setting where very few conventional $\mathrm{T}$ cells are transplanted). ${ }^{16,22,31,32}$ Some groups of investigators are focusing their research on ex vivo Treg expansion. ${ }^{23,31}$ While this approach could yield sufficient Treg numbers with very good purity when mTor inhibitors are added to the culture media, the fate of ex vivo expanded Treg after infusion in humans has remained poorly understood, although Brunstein and colleagues ${ }^{23}$ could detect ex vivo expanded (without mTor inhibition) Treg in the PB up to 14 days after infusion in the context of unrelated cord blood transplantation. Further potential limitations of Treg ex vivo expansion include reduction in their T-cell repertoire, as well as possible expansion of Th17 cells when ex vivo expansion is carried out without mTor inhibitors. ${ }^{33}$ In addition, given that mTor inhibitors also induce FoxP3 on conventional T cells, ${ }^{34}$ Treg ex vivo expansion in the presence of mTor inhibitors does not exclude the risk of a contamination by induced Treg that could revert in vivo, in the absence of in vivo mTor inhibition. ${ }^{31}$ Thus, another approach might consist of infusing a less pure population of freshly isolated Treg (obtained by clinical-grade immunomagnetic selection), ${ }^{35,36}$ followed by in vivo selection or expansion with sirolimus and/or low-dose IL-2, ${ }^{37,38}$ thus avoiding the need for culturing Treg ex vivo in good manufacturing practice conditions. However, before proposing this easier approach to patients with steroid-refractory chronic GVHD, we wanted to investigate the ability of clinical-grade enriched Treg to attenuate experimental xenogeneic GVHD induced by PBMNC (autologous to Treg) infusion in NSG mice. Given that the purity of the enriched fraction was not $100 \%$, it was also necessary to verify their inability to induce xenogeneic GVHD when infused alone. Several observations were made.

First, this report confirms the chosen combined negative and positive separation strategy to select Treg performed by using the CliniMACS plus system (device and reagents) allows recovery of a large number of enriched CD4+CD25+FoxP3+ $\mathrm{T}$ cells, with a median purity ranging from $42 \%$ to $68 \%$. This is in line with what was observed by other groups of investigators. ${ }^{22}$ Interestingly, we also demonstrated in sorted cells from Donor 5 that naive Treg (in comparison to memory Treg) were markedly enriched for recent thymus emigrants expressing more than 20 times more TRECs per $10^{5}$ cells.

Second and more importantly, coinfusion of enriched Treg at a PBMNC/Treg-enriched ratio of 2/1 (with a real non-Treg/Treg [defined as $\mathrm{CD} 4+\mathrm{CD} 25^{\text {high }}$ FoxP3+] ratio ranging from 2.5 to 3.3) delayed the development of xenogeneic GVHD and prolonged survival. These results are in line with two previous reports demonstrating that coinfusion of fresh CD25+ T cells or of expanded Treg (with a purity of $70 \%$ ) attenuated xenogeneic GVHD caused by infusion of PBMNCs (autologous to Treg) in another deficient mouse strain (Rag2-/-gammaC-/- mice). ${ }^{39,40}$ Our results are also in line with those observed by Hippen and colleagues $^{41}$ and by Chakraborty and colleagues ${ }^{42}$ who demonstrated that coinfusion of expanded Treg at a PBMNC/Treg ratio of 1 / 1 decreased xenogeneic GVHD and increased survival of NSG mice. The originality of our work 


\begin{tabular}{|c|c|c|c|c|c|c|c|c|c|c|c|c|}
\hline \multirow{2}{*}{$\begin{array}{l}\text { Histologic findings } \\
\text { Experiment (one mouse/experiment/group) }\end{array}$} & \multicolumn{4}{|c|}{ PBMNC only } & \multicolumn{4}{|c|}{ PBMNC + Treg } & \multicolumn{4}{|c|}{ Treg only } \\
\hline & 2 & 3 & 5 & 6 & 2 & 3 & 5 & 6 & 2 & 3 & 5 & 6 \\
\hline Perivascular lymphocytic infiltrate & 2 & 3 & 3 & 3 & 2 & 3 & 4 & 1 & 3 & 0 & 0.5 & 0 \\
\hline Interstitial lymphocytic infiltrate & 1 & 3 & 2 & 1 & 0.5 & 3 & 2 & 2 & 2 & 0 & 0.5 & 0 \\
\hline Peribronchiolar lymphocytic infiltrate & 1 & 3 & 3 & 1 & 0.5 & 2 & 4 & 3 & 2 & 0 & 0.5 & 0 \\
\hline Endothelitis & 3 & 2 & 0 & 0 & 1 & 3 & 3 & 0 & 0 & 0 & 0 & 0 \\
\hline Bronchial epithelial apoptosis & 0.5 & 2 & 0.5 & 0.5 & 1 & 1 & 3 & 1 & 1 & 0 & 0.5 & 0 \\
\hline Bronchial epithelial detachment & 0.5 & 1 & 1 & 0.5 & 0.5 & 0.5 & 3 & 0.5 & 0 & 0 & 0 & 0 \\
\hline Alveolar edema & 0 & 0 & 0 & 0 & 0 & 0 & 0 & 0 & 0 & 0 & 0 & 0 \\
\hline Alveolar debris & 0 & 0.5 & 0.5 & 0 & 0 & 0 & 2 & 0.5 & 0 & 0 & 0 & 0 \\
\hline Alveolar damage & 0 & 0 & 0 & 0 & 0 & 0 & 0 & 0 & 0 & 0 & 0 & 0 \\
\hline Total score & 8 & 14.5 & 10 & 6 & 5.5 & 12.5 & 21 & 8 & 8 & 0 & 2 & 0 \\
\hline Total score without infiltration criteria & 4 & 5.5 & 2 & 1 & 1.5 & 4.5 & 11 & 2 & 1 & 0 & 0.5 & 0 \\
\hline
\end{tabular}

* In this semiquantitative scoring system $0=$ normal, $0.5=$ focal and rare, $1=$ focal and mild, $2=$ diffuse and mild, $3=$ diffuse and moderate, and 4 = diffuse and severe.

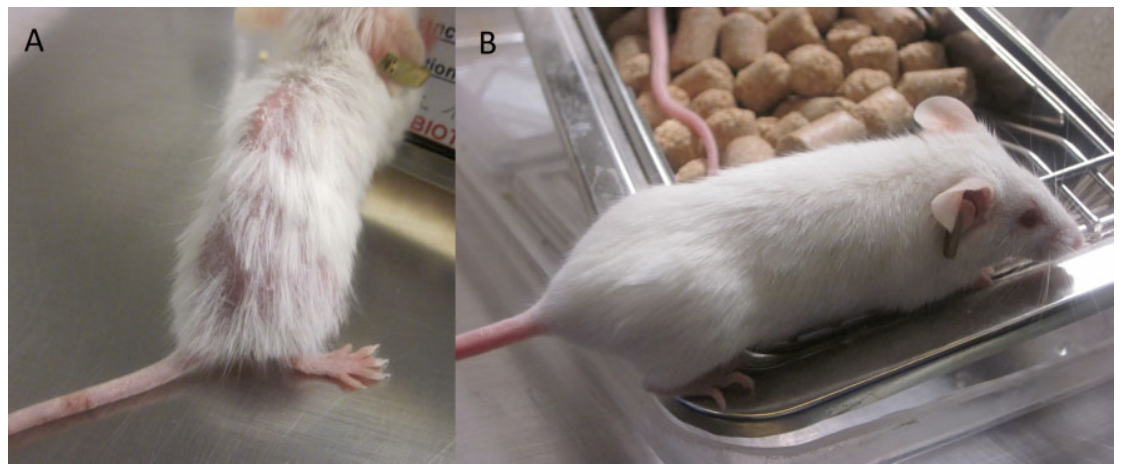

Fig. 3. (A) Representative picture of a NSG mouse suffering from xenogeneic chronic GVHD 50 days after receiving PBMNC + Treg infusion. (B) Representative picture of a NSG mouse 106 days after receiving Treg-enriched cells only.

in comparison with those previous reports is that we used freshly isolated Treg (using clinical-grade compliant method) and not expanded Treg. Although Treg-enriched infusion attenuated xenogeneic GVHD in our study, they only provided a temporary protection from xenogeneic GVHD since most mice who survived beyond Day 75 after PBMNC/Treg coinfusion eventually developed signs of "xenogeneic chronic GVHD" (skin fibrosis and hair loss [Fig. 3]). This could be due to a larger expansion of conventional $\mathrm{T}$ cells compared to that of Treg in our model (in line with the apparent disappearance of Treg when infused alone in NSG mice) or to the fact that we used a suboptimal ratio of PBMNC/Treg (for example the PBMNC/Treg ratio in the study by Di Ianni and coworkers ${ }^{22}$ was $1 / 2$ instead of $2 / 1$ in current study). Further studies are needed to assess whether approaches combining Treg infusion with rapamycin or low-dose IL-2 will allow to totally abrogate xenogeneic GVHD in this model and to assess the impact of Treg infusion on engraftment of human hematopoietic stem/ progenitor cells in NSG mice.

Third, infusion of the Treg-enriched fraction alone did not induce xenogeneic GVHD, even in the sixth cohort where Treg purity was only $42 \%$ and mice thus received more than $1 \times 10^{6}$ conventional CD4+ T cells. Interestingly, cells from Treg-enriched fractions when infused alone not only did not cause GVHD, but also did not expand (or if they expanded underwent apoptosis), as shown by the very low human cell infiltration in NSG mice observed at necropsy. A probable explanation is that Treg inhibited in vivo the conventional activated CD4+ T cells contained in the Treg-enriched fraction, while true Treg did not expand probably due to a lack of human IL-2 in that model when only enriched Treg are infused, as observed in a recent report from Abraham and colleagues. ${ }^{43}$ Further, clinical studies have demonstrated that, after allo-HCT, infusion of CD8-depleted donor lymphocytes induced less acute GVHD than infusion of unmanipulated DLI, ${ }^{44}$ suggesting that $\mathrm{CD} 4+\mathrm{T}$ cells were less prone to induce GVHD than CD8+ T cells in humans.

In summary, our data suggest that infusion of clinicalgrade enriched Treg delayed the occurrence of xenogeneic GVHD induced by human autologous PBMNCs (at a PBMNC/enriched Treg ratio of 2/1) in NSG mice, at least when Treg purity in the Treg-enriched fraction was more than $55 \%$. Based on these results, a pilot study of CliniMACS-enriched donor Treg infusion together with in vivo selection with rapamycin is under development as treatment for patients with steroid-refractory chronic GVHD.

\section{ACKNOWLEDGMENTS}

We are grateful to Sophie Dubois for help with mouse experiments and to Sandra Ormenese from the Imaging and Flow Cytometry Platform of the GIGA for help with flow cytometry analyses. The authors are also grateful to Nadine Wanten, 
A

Fig. 4. (A) Survival of NSG mice given $2 \times 10^{6}$ enriched human Treg alone after 2.5 Gy TBI the day before transplantation $(n=21)$. Mice scheduled to be euthanized around Day 26 (Days 25-27) for flow cytometry analyses were censored on the graft. (B) Proportion of CD4+ T cells positive for FoxP3 around Day 26 (Days 25-27) after transplantation in NSG mice given $2 \times 10^{6}$ enriched human Treg only after 2.5 Gy TBI the day before transplantation. Horizontal lines represent median values.

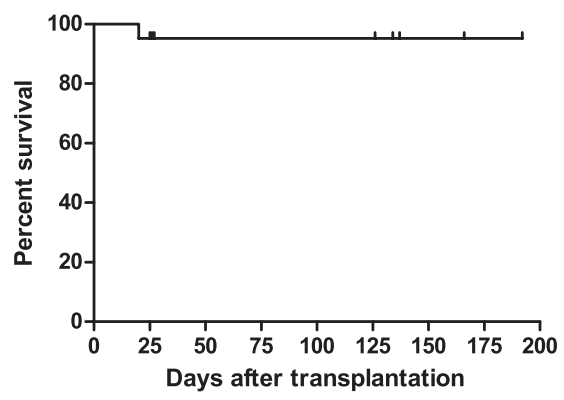

with nonmyeloablative conditioning. J Clin Oncol 2005;23:1993-2003.

5. Baron F, Labopin M, Niederwieser D, Vigouroux S, Cornelissen JJ, Malm C, Vindelov LL, Blaise D, Janssen JJ, Petersen E, Socié G, Nagler A, Rocha V, Mohty M. Impact of graft-versus-host disease after reduced-intensity conditioning allogeneic stem cell transplantation for acute myeloid leukemia: a report from the Acute Leukemia Working Party of the European group for blood and marrow transplantation. Leukemia 2012;26:2462-8.

6. Socie G, Blazar BR. Acute graft-versushost disease: from the bench to the bedside. Blood 2009;114:4327-36.

7. Blazar BR, Murphy WJ, Abedi M. Advances in graft-versus-host disease biology and therapy. Nat Rev Immunol 2012;12:443-58.

Sylvianne Simar, Olivier Dengis, Amélie Halleux, and Coline Daulne for excellent technical assistance. We are grateful to Miltenyi Biotec $\mathrm{GmbH}$ for providing us with columns and antibodies. Study design, FBa and SHB; data analyses, MH, CL, LS, and FBa; donor recruitment, CL and EB; donor evaluation, FBa; Treg collection and enrichment, $\mathrm{CL}, \mathrm{EB}, \mathrm{OG}, \mathrm{MC}$, and $\mathrm{YB}$; mice experiments, MH, LB, FBr, and PD; TREC analyses, MH; flow cytometry analyses, MH, LB, and SHB; FOXP3i1 sequences methylation, SL; histology, JS and PD; manuscript writing, FBa, MH, and SHB; manuscript editing, CL, SL, EB, PhD, PiD, and YB; and approval of the manuscript, all authors.

\section{CONFLICT OF INTEREST}

The authors have no conflict of interest.

\section{REFERENCES}

1. Baron F, Storb R. Allogeneic hematopoietic cell transplantation as treatment for hematological malignancies: a review. Springer Semin Immunopathol 2004;26:71-94.

2. Weiden PL, Sullivan KM, Flournoy N, Storb R, Thomas ED; the Seattle Marrow Transplant Team. Antileukemic effect of chronic graft-versus-host disease. Contribution to improved survival after allogeneic marrow transplantation. N Engl J Med 1981;304:1529-33.

3. Riddell SR, Berger C, Murata M, Randolph S, Warren EH. The graft versus leukemia response after allogeneic hematopoietic stem cell transplantation. Blood Rev 2003; 17:153-62.

4. Baron F, Maris MB, Sandmaier BM, Storer BE, Sorror M, Diaconescu R, Woolfrey AE, Chauncey TR, Flowers ME, Mielcarek M, Maloney DG, Storb R. Graft-versus-tumor effects after allogeneic hematopoietic cell transplantation M, Weisdorf DJ, Flowers ME, Martin PJ, Palmer J, Jacobsohn D, Pavletic SZ, Vogelsang GB, Lee SJ. Global and organ-specific chronic graft-versus-host disease severity according to the 2005 NIH Consensus Criteria. Blood 2011; 118:4242-9.

9. Thepot S, Zhou J, Perrot A, Robin M, Xhaard A, de Latour RP, Ades L, Ribaud P, Petropoulou AD, Porcher R, Socié G. The graft-versus-leukemia effect is mainly restricted to NIH-defined chronic graft-versus-host disease after reduced intensity conditioning before allogeneic stem cell transplantation. Leukemia 2010;24:1852-8.

10. Weiden PL, Storb R, Tsoi MS, Graham TC, Lerner KG, Thomas ED. Infusion of donor lymphocytes into stable canine radiation chimeras: implications for mechanism of transplantation tolerance. J Immunol 1976;116:1212-9.

11. Sakaguchi S, Sakaguchi N, Shimizu J, Yamazaki S, Sakihama T, Itoh M, Kuniyasu Y, Nomura T, Toda M, Takahashi T. Immunologic tolerance maintained by CD25+ CD4+ regulatory $\mathrm{T}$ cells: their common role in controlling autoimmunity, tumor immunity, and transplantation tolerance [Review]. Immunol Rev 2001;182:18-32.

12. Tian L, Humblet-Baron S, Liston A. Immune tolerance: are regulatory $\mathrm{T}$ cell subsets needed to explain suppression of autoimmunity? Bioessays 2012;34:569-75.

13. Bennett CL, Christie J, Ramsdell F, Brunkow ME, Ferguson PJ, Whitesell L, Kelly TE, Saulsbury FT, Chance PF, Ochs HD. The immune dysregulation, polyendocrinopathy, enteropathy, X-linked syndrome (IPEX) is caused by mutations of FOXP3. Nat Genet 2001;27:20-1.

14. Cohen JL, Trenado A, Vasey D, Klatzmann D, Salomon BL. CD4(+)CD25(+) immunoregulatory T cells: new therapeutics for graft-versus-host disease. J Exp Med 2002;196: 401-6.
8. Arai S, Jagasia M, Storer B, Chai X, Pidala J, Cutler C, Arora 
15. Edinger M, Hoffmann P, Ermann J, Drago K, Fathman CG, Strober S, Negrin RS. CD4+CD25+ regulatory T cells preserve graft-versus-tumor activity while inhibiting graftversus-host disease after bone marrow transplantation. Nat Med 2003;9:1144-50.

16. Colonna L, Sega EI, Negrin RS. Natural and expanded CD4(+)CD25(+) regulatory T cells in bone marrow transplantation. Biol Blood Marrow Transplant 2011;17(1 Suppl):S58-S62.

17. Matsuoka K, Kim HT, McDonough S, Bascug G, Warshauer B, Koreth J, Cutler C, Ho VT, Alyea EP, Antin JH, Soiffer RJ, Ritz J. Altered regulatory $\mathrm{T}$ cell homeostasis in patients with CD4+ lymphopenia following allogeneic hematopoietic stem cell transplantation. J Clin Invest 2010;120:147993.

18. Kawano Y, Kim HT, Matsuoka K, Bascug G, McDonough S, Ho VT, Cutler C, Koreth J, Alyea EP, Antin JH, Soiffer RJ, Ritz J. Low telomerase activity in CD4+ regulatory T cells in patients with severe chronic GVHD after hematopoietic stem cell transplantation. Blood 2011;118:5021-30.

19. Lord JD, Hackman RC, Gooley TA, Wood BL, Moklebust AC, Hockenbery DM, Steinbach G, Ziegler SF, McDonald GB. Blood and gastric FOXP3+ T cells are not decreased in human gastric graft-versus-host disease. Biol Blood Marrow Transplant 2011;17:486-96.

20. Rieger K, Loddenkemper C, Maul J, Fietz T, Wolff D, Terpe H, Steiner B, Berg E, Miehlke S, Bornhauser M, Schneider T, Zeitz M, Stein H, Thiel E, Duchmann R, Uharek L. Mucosal FOXP3+ regulatory T cells are numerically deficient in acute and chronic GvHD. Blood 2006;107: 1717-23.

21. Magenau JM, Qin X, Tawara I, Rogers CE, Kitko C, Schlough M, Bickley D, Braun TM, Jang PS, Lowler KP, Jones DM, Choi SW, Reddy P, Mineishi S, Levine JE, Ferrara JL, Paczesny S. Frequency of CD4(+)CD25(hi)FOXP3(+) regulatory $\mathrm{T}$ cells has diagnostic and prognostic value as a biomarker for acute graft-versus-host-disease. Biol Blood Marrow Transplant 2010;16:907-14.

22. Di Ianni M, Falzetti F, Carotti A, Terenzi A, Castellino F, Bonifacio E, Del Papa B, Zei T, Ostini RI, Cecchini D, Aloisi T, Perruccio K, Ruggeri L, Balucani C, Pierini A, Sportoletti P, Aristei C, Falini B, Reisner Y, Velardi A, Aversa F, Martelli MF. Tregs prevent GVHD and promote immune reconstitution in HLA-haploidentical transplantation. Blood 2011; 117:3921-8.

23. Brunstein CG, Miller JS, Cao Q, McKenna DH, Hippen KL, Curtsinger J, DeFor T, Levine BL, June CH, Rubinstein P, McGlave PB, Blazar BR, Wagner JE. Infusion of ex vivo expanded $\mathrm{T}$ regulatory cells in adults transplanted with umbilical cord blood: safety profile and detection kinetics. Blood 2011;117:1061-70.

24. Bruck F, Belle L, Lechanteur C, de Leval L, Hannon M, Dubois S, Castermans E, Humblet-Baron S, Rahmouni S, Beguin Y, Briquet A, Baron F. Impact of bone marrowderived mesenchymal stromal cells on experimental xenogeneic graft-versus-host disease. Cytotherapy 2013;15: 267-79.

25. Canavan JB, Afzali B, Scotta C, Fazekasova H, Edozie FC, Macdonald TT, Hernandez-Fuentes MP, Lombardi G, Lord GM. A rapid diagnostic test for human regulatory T-cell function to enable regulatory T-cell therapy. Blood 2012; 119:e57-e66.

26. Castermans E, Baron F, Willems E, Schaaf-Lafontaine N, Meuris N, Gothot A, Vanbellighen JF, Herens C, Seidel L, Geenen V, Cheynier R, Beguin Y. Evidence for neogeneration of $\mathrm{T}$ cells by the thymus after nonmyeloablative conditioning. Haematologica 2008;93:240-7.

27. Castermans E, Hannon M, Dutrieux J, Humblet-Baron S, Seidel L, Cheynier R, Willems E, Gothot A, Vanbellinghen JF, Geenen V, Sandmaier BM, Storb R, Beguin Y, Baron F. Thymic recovery after allogeneic hematopoietic cell transplantation with non-myeloablative conditioning is limited to patients younger than 60 years of age. Haematologica 2011;96:298-306.

28. Stockis J, Fink W, Francois V, Connerotte T, de Smet C, Knoops L, van der Bruggen P, Boon T, Coulie PG, Lucas S. Comparison of stable human Treg and Th clones by transcriptional profiling. Eur J Immunol 2009;39:869-82.

29. Lucas S, van Baren N, de Smet C, Coulie PG. Demethylation of the FOXP3 gene in human melanoma cells precludes the use of this epigenetic mark for quantification of Tregs in unseparated melanoma samples. Int J Cancer 2012;130:1960-6.

30. Nervi B, Rettig MP, Ritchey JK, Wang HL, Bauer G, Walker J, Bonyhadi ML, Berenson RJ, Prior JL, Piwnica-Worms D, Nolta JA, DiPersio JF. Factors affecting human T cell engraftment, trafficking, and associated xenogeneic graftvs-host disease in NOD/SCID beta2mnull mice. Exp Hematol 2007;35:1823-38.

31. Edinger M, Hoffmann P. Regulatory T cells in stem cell transplantation: strategies and first clinical experiences. Curr Opin Immunol 2011;23:679-84.

32. Humblet-Baron S, Baron F, Liston A. Regulatory T cells fulfil their promise? Immunol Cell Biol 2011;89:825-6.

33. Tresoldi E, Dell'Albani I, Stabilini A, Jofra T, Valle A, Gagliani N, Bondanza A, Roncarolo MG, Battaglia M. Stability of human rapamycin-expanded CD4+CD25+ T regulatory cells. Haematologica 2011;96:1357-65.

34. Long SA, Buckner JH. Combination of rapamycin and IL-2 increases de novo induction of human CD4(+)CD25(+)FOXP3(+) T cells. J Autoimmun 2008;30: 293-302.

35. Hoffmann P, Boeld TJ, Eder R, Albrecht J, Doser K, Piseshka B, Dada A, Niemand C, Assenmacher M, Orso E, Andreesen R, Holler E, Edinger M. Isolation of CD4+CD25+ regulatory $\mathrm{T}$ cells for clinical trials. Biol Blood Marrow Transplant 2006;12:267-74.

36. Di IM, Del PB, Cecchini D, Bonifacio E, Moretti L, Zei T, Ostini RI, Falzetti F, Fontana L, Tagliapietra G, Maldini C, Martelli MF, Tabilio A. Immunomagnetic isolation of 
CD4+CD25+FoxP3+ natural T regulatory lymphocytes for clinical applications. Clin Exp Immunol 2009;156: 246-53.

37. Shin HJ, Baker J, Leveson-Gower DB, Smith AT, Sega EI, Negrin RS. Rapamycin and IL-2 reduce lethal acute graftversus-host disease associated with increased expansion of donor type CD4+CD25+Foxp3+ regulatory T cells. Blood 2011;118:2342-50.

38. Koreth J, Matsuoka K, Kim HT, McDonough SM, Bindra B, Alyea EP, III, Armand P, Cutler C, Ho VT, Treister NS, Bienfang DC, Prasad S, Tzachanis D, Joyce RM, Avigan DE, Antin JH, Ritz J, Soiffer RJ. Interleukin-2 and regulatory T cells in graft-versus-host disease. N Engl J Med 2011;365: 2055-66.

39. Mutis T, van Rijn RS, Simonetti ER, Aarts-Riemens T, Emmelot ME, van Bloois L, Martens A, Verdonck LF, Ebeling SB. Human regulatory T cells control xenogeneic graft-versus-host disease induced by autologous $\mathrm{T}$ cells in RAG2-/-gammac-/- immunodeficient mice. Clin Cancer Res 2006;12:5520-5.

40. Vercoulen Y, Guichelaar T, Meerding J, Emmelot M, Pingen M, Storm G, Coffer P, Sawitzki B, Martens A, Mutis T, Prakken B. Application of cultured human regulatory $\mathrm{T}$ cells requires preclinical in vivo evaluation. J Allergy Clin Immunol 2012;129:852-5.

41. Hippen KL, Merkel SC, Schirm DK, Sieben CM, Sumstad D, Kadidlo DM, McKenna DH, Bromberg JS, Levine BL, Riley JL, June CH, Scheinberg P, Douek DC, Miller JS, Wagner JE, Blazar BR. Massive ex vivo expansion of human natural regulatory T cells (T(regs)) with minimal loss of in vivo functional activity. Sci Transl Med 2011;3:83ra41.

42. Chakraborty R, Mahendravada A, Perna SK, Rooney CM, Heslop HE, Vera JF, Savoldo B, Dotti G. Robust and cost effective expansion of human regulatory $\mathrm{T}$ cells highly functional in a xenograft model of graft versus host disease. Haematologica 2012;98:533-7.

43. Abraham S, Pahwa R, Ye C, Choi JG, Pahwa S, Jaggaiahgari S, Raut A, Chen S, Manjunath N, Shankar P. Long-term engraftment of human natural T regulatory cells in NOD/ SCID IL2rgammac(null) mice by expression of human IL-2. Plos ONE 2012; 7:e51832.

44. Soiffer RJ, Alyea EP, Hochberg E, Wu C, Canning C, Parikh B, Zahrieh D, Webb I, Antin J, Ritz J. Randomized trial of CD8+ T-cell depletion in the prevention of graft-versushost disease associated with donor lymphocyte infusion. Biol Blood Marrow Transplant 2002;8:625-32. 